\title{
SELF-DUALITY EQUATIONS FOR SPHERICALLY SYMMETRIC SU(2) GAUGE FIELDS
}

\author{
Zet Gheorghe \\ Department of Physics, Technical University "Gh.Asachi" \\ Iasi, Romania
}

May 14, 2001

\begin{abstract}
A model of spherically symmetric $\mathrm{SU}(2)$ gauge theory is considered. The self-duality equations are written and it is shown that they are compatible with the Einstein-Yang-Mills equations.It is proven that this property is true for any gauge theory with curved base spacetime and having a compact Lie group as structural group.
\end{abstract}

\section{Introduction}

The self-duality equations are important in gauge theories because they show the connection between gauge models with internal symmetry groups and gauge theory of gravity. They are differential equations of the first order and it is easier to investigate the solutions for different particular configurations of the gauge fields and of space-times. One of the most important property of the self-duality equations is that they imply the Yang-Mills field equations. In this paper we will prove this property for the general case of a gauge theory with compact Lie group of symmetry over a 4-dimensional space-time manifold.

It is important to remark that there are $3 m$ independent self-duality equations (of the first order) while the number of Yang-Mills equations is 
equal to $4 m$, where $m$ is the dimension of the gauge group.Both of them have $4 m$ unknown functions which are the gauge potentials $A_{\mu}^{a}(x), a=1,2, \ldots, m$; $\mu=0,1,2,3$. But, we have, in addition, $m$ gauge conditions for $A_{\mu}^{a}(x)$ (for example Coulomb, Lorentz or axial gauge) which together with the selfduality equation constitute a system of $4 m$ equations. The Bianchi identities for the self-dual stress tensor $F_{\mu \nu}^{a}$ coincide with the Yang-Mills equations and do not imply therefore suplementary conditions.

In the sections 2 and 3 we will use the axial gauge in order to obtain the self duality equations for a $S U(2)$ gauge theory over a cureved space-time. The section 4 is devoted to the study of compatibility between self-duality and Yang-Mills equations.In fact, we will write the Einstein-Yang-Mills equations and we will analyse only the Yang-Mills sector. The Einstein equations can not be obtained of course from self-duality. They should be obtained if we would consider a gauge theory having $P \times S U(2)$ as symmetry group, where $P$ is the Poincaré group. More generally, a gauge theory of $\mathrm{N}$-extended supersymmetry can be developed by imposing the self-duality condition.

\section{The field-strength tensor of the gauge fields}

We will consider a $S U(2)$ gauge theory over a 4-dimensional space-time manifold $M$, spherically symmetric, endowed with the metric:

$$
d s^{2}=\sigma^{2} N d t^{2}-\frac{1}{N} d r^{2}-r^{2}\left(d \theta^{2}+\sin ^{2} \theta d \varphi^{2}\right),
$$

where $\sigma$ and $N$ are function depending only of $r$ variable. For $\sigma=1$ and $N=1-\frac{2 m}{r}$ we obtain the Schwarzschild metric, while for $\sigma=1$ and $N=$ $1-\frac{2 M}{r}+\frac{Q^{2}+1}{r^{2}}$ we have the Reissner-Nordström (RS) metric. The components of the metric tensor $g_{\mu \nu}$ are:

$$
g_{00}=\sigma^{2} N, g_{11}=-\frac{1}{N}, g_{22}=-r^{2}, g_{33}=-r^{2} \sin ^{2} \theta,
$$

and the determinant of this tensor is

$$
g=\operatorname{det}\left(g_{\mu \nu}\right)=-\sigma^{2} r^{4} \sin ^{2} \theta, \sqrt{-g}=\sigma r^{2} \sin \theta .
$$

The Lie algebra of $S U(2)$ group is characterized by the structure equations:

$$
\left[T_{a}, T_{b}\right]=\varepsilon_{a b c} T_{c} ; a, b, c=1,2,3
$$


where $\varepsilon_{a b c}$ is the Levi-Civita symbol of rank 3 with $\varepsilon_{123}=1$. The basic elements of the model are $\left(M, g_{\mu \nu}, A\right)$, where $M$ is the space-time manifold with the metric coefficients given in (2.2), and the Lie algebra-valued 1-form is $A=A_{\mu} d x^{\mu}=A_{\mu}^{a} T_{a} d x^{\mu}$. The Lie algebra-valued 2-form of the gauge fields $A_{\mu}^{a}$ is defined by:

$$
F=\frac{1}{2} F_{\mu \nu} d x^{\mu} \wedge d x^{\nu}=\frac{1}{2} F_{\mu \nu}^{a} T_{a} d x^{\mu} \wedge d x^{\nu},
$$

where $F_{\mu \nu}^{a}$ are the components of the stress tensor:

$$
F_{\mu \nu}^{a}=\partial_{\mu} A_{\nu}^{a}-\partial_{\nu} A_{\mu}^{a}+\varepsilon_{a b c} A_{\mu}^{b} A_{\nu}^{c}
$$

The spherically symmetric $S U(2)$ gauge fields will be parametrized as $(\text { Witten ansatz })^{1}$ :

$$
A=u T_{3} d t+w\left(T_{2} d \theta-T_{1} \sin \theta d \varphi\right)+T_{3} \cos \theta d \varphi,
$$

where $u$ and $w$ are function depending only of variable $r$. Using the ansatz (2.7), we obtain the following non-null components of the stress tensor:

$$
\begin{aligned}
& F_{02}^{1}=-u w, F_{13}^{1}=-w^{\prime} \sin \theta, \\
& F_{03}^{2}=-u w \sin \theta, F_{12}^{2}=w^{\prime}, \\
& F_{03}^{3}=-u^{\prime}, F_{23}^{3}=\left(w^{2}-1\right) \sin \theta,
\end{aligned}
$$

with $u^{\prime}=\frac{d u}{d r}$ and $w^{\prime}=\frac{d w}{d r}$.

Now, we introduce the dual 2-form $* F$ (the symbol " $*$ denoting the Hodge dual map) whose components are defined by:

$$
* F_{\mu \nu}^{a}=\frac{1}{2} \sqrt{-g} \varepsilon_{\mu \nu \rho \lambda} F^{a \rho \lambda},
$$

where $\varepsilon_{\mu \nu \rho \lambda}$ is the Levi-Civita symbol of rank 4 with $\varepsilon_{0123}=1$. The non-null components of $* F$ are:

$$
\begin{aligned}
& * F_{02}^{1}=\sigma N w^{\prime}, * F_{13}^{1}=-\frac{u w \sin \theta}{\sigma N}, \\
& * F_{03}^{2}=\sigma N w^{\prime} \sin \theta, * F_{12}^{2}=\frac{u w}{\sigma N}, \\
& * F_{01}^{3}=\frac{\sigma\left(w^{2}-1\right)}{r^{2}}, * F_{23}^{3}=\frac{r^{2} u^{\prime} \sin \theta}{\sigma} .
\end{aligned}
$$


In the next section we will impose the self-duality condition $* F=i F$ for the 2-form $F$ and we will obtain the self-duality equations for the $S U(2)$ gauge fields. We will show that these equations are compatible with the EinsteinYang-Mills $(E Y M)$ equations of the model. In fact, we will prove that this property is true for any self-dual gauge theory with compact gauge Lie group.

\section{Self-duality equations}

A self-dual (or anti-self-dual) form $T$ over a differential manifold $M$ can be constructed only if $M$ is of even dimension and the following equation is satisfied $^{2}$ :

$$
* * T=\lambda T ; \operatorname{rankT}=\frac{1}{2} \operatorname{dim} M .
$$

But, the dual map (or the Hodge-duality) has the property:

$$
\begin{aligned}
* * T & =(-1)^{k(n-k)} T(\text { for Euclidean metric }), \\
* * T & =-(-1)^{k(n-k)} T(\text { for Minkowski metric }),
\end{aligned}
$$

where $k$ is the rank of $T$ and $n$ is the dimension of $M$. This means that the quantity $\lambda$ in (3.1) is constrained to very special values:

$$
\pm T=* * T=*(\lambda T)=\lambda^{2} T
$$

that is

$$
\begin{aligned}
& \lambda= \pm 1, \text { if } * * T=T, \text { (Euclidean metric) }, \\
& \lambda= \pm i, \text { if } * * T=-T, \text { (Minkowski metric). }
\end{aligned}
$$

In our model, the rank of $F$ is $k=2$ and the dimension of the space-time $M$ is $n=4$. Then, the self-duality condition is ${ }^{3}$ :

$$
* F=i F
$$

Now, if we introduce the components (2.8) and (2.10) in (3.4), we obtain the following self-duality equations:

$$
\begin{aligned}
\sigma N w^{\prime} & =-i u w \\
\frac{r^{2}}{\sigma} u^{\prime} & =i\left(w^{2}-1\right) .
\end{aligned}
$$


These equations are of the first order and they determine the gauge potential functions $u(r)$ and $w(r)$. The metric coefficients $\sigma$ and $N$ are determined by the EYM equations which are of second order and, as we will prove in the next section, they determine also the gauge potential functions $u(r)$ and $w(r)$.

The simplest solution of the self-duality equations (3.5) coupled with Einstein equations (see Sect.4) is ${ }^{1}$ :

$$
u=0, w= \pm 1, \sigma=1, N=1-\frac{2 m}{r},
$$

which describes the Schwarzschild metric and a pure gauge Yang-Mills field. The simplest solution with a non-trivial gauge field describes colored black holes ${ }^{4}$ :

$$
u=u(\infty)+\frac{Q}{r}, w=0, N=1-\frac{2 M}{r}+\frac{Q^{2}+1}{r^{2}}, \sigma=1 .
$$

It corresponds to the Reissner-Nordström (RN) metric with the electric charge $Q$ and the unit magnetic charge. Many others solutions (particlelike, sphaleron type, with $\Lambda$-term, stringy type, axially symmetric etc.) are given by Volkov and Gal'tsov ${ }^{1}$. Local solutions of the static, spherically symmetric, EYM equations with $S U(2)$ gauge group are studied by Zotov ${ }^{5}$ on the basis of dynamical system methods. In this case it is proven the existence of solutions with oscillating metric as well as the existence of local solutions for all known formal series expansions. Exact solutions for self-dual SU(2) gauge theory with axial symmetry are given in our paper ${ }^{6}$.

\section{Einstein-Yang-Mills equations}

The integral action of our model is:

$$
S_{E Y M}=\int\left\{-\frac{1}{16 \pi G} R-\frac{1}{4 K e^{2}} \operatorname{Tr} F_{\mu \nu} F^{\mu \nu}\right\} \sqrt{-g} d^{4} x,
$$

where $e$ is the gauge coupling constant, $R$ is the scalar curvature associated to $g_{\mu \nu}$ and $\operatorname{Tr}\left(T_{a} T_{b}\right)=K \delta_{a b}$. For $G=S U(2)$ we choose $T_{a}=\frac{1}{2} \tau_{a}\left(\tau_{a}\right.$ being the Pauli matrices) and then $K=\frac{1}{2}$. The gravitational constant $G$ is the only dimensionful quantity in the action (the units $\hbar=c=1$ are understood).

Taking $\delta S_{E Y M}=0$ with respect to $A_{\mu}^{a}$ and $g_{\mu \nu}$ fields, we obtain the following general form of the EYM equations ${ }^{3}$ :

$$
\frac{1}{\sqrt{-g}} \partial_{\mu}\left(\sqrt{-g} F^{a \mu \nu}\right)+f_{b c}^{a} A_{\mu}^{b} F^{c \mu \nu}=0,(\text { Yang }- \text { Mills equations }),
$$


where $f_{b c}^{a}=-f_{c b}^{a}$ are the structure constants of the gauge group, and respectively

$$
R_{\mu \nu}-\frac{1}{2} g_{\mu \nu}=8 \pi G T_{\mu \nu},(\text { Ei nstein equations }),
$$

with the gauge-invariant stress-energy tensor

$$
T_{\mu \nu}=\frac{1}{K e^{2}} \operatorname{Tr}\left(-F_{\mu \rho} F_{\nu}^{\rho}+\frac{1}{4} F_{\rho \lambda} F^{\rho \lambda}\right),
$$

Then, introducing (2.2) and (2.8) in (4.2) and (4.3) we obtain the EYM equations of our model:

$$
\begin{gathered}
\left(\sigma N w^{\prime}\right)^{\prime}=\frac{\sigma}{r^{2}} w\left(w^{2}-1\right)-\frac{1}{\sigma N} u^{2} w \\
\left(\frac{r^{2}}{\sigma} u^{\prime}\right)^{\prime}=\frac{2}{\sigma N} u w^{2} \\
\frac{\sigma^{\prime}}{\sigma}=\frac{2}{r} w^{\prime 2}+\frac{2}{N^{2} \sigma^{2} r} w^{2} u^{2} \\
\frac{1}{2}\left(N^{\prime} r+N-1\right)+\frac{r^{2}}{2 \sigma^{2}} u^{\prime 2}+\frac{1}{N \sigma^{2}} u^{2} w^{2}+N w^{\prime 2}+\frac{1}{2 r^{2}}\left(w^{2}-1\right)^{2}=0
\end{gathered}
$$

where we used $K=\frac{1}{2}$ and $\frac{4 \pi G}{e^{2}}=1$ units. These equations admit the particular solutions given in (3.6) and (3.7).

Let us compare the self-duality equations (3.5) with the first two EYM equations (4.5) and (4.6). If we take the derivatives with respect to $r$ of the equations (3.5), then we obtain:

$$
\begin{aligned}
& \left(\sigma N w^{\prime}\right)^{\prime}=-i\left(u^{\prime} w+u w^{\prime}\right), \\
& \left(\frac{r^{2}}{\sigma} u^{\prime}\right)^{\prime}=2 i w w^{\prime} .
\end{aligned}
$$

Now, if we replace $i w^{\prime}$ and $i u^{\prime}$ deduced from (3.5) into the left-hand sides of (4.9), then we obtain the EYM equations (4.5) and (4.6). Of course, the other two EYM equations (4.7) and (4.8) can not be obtained from the selfduality equations of the gauge fields. This may be possible if we develope a gauge theory with the gauge group $P \times S U(2)$, where $P$ is the Poincaré group $^{3}$. 
In fact, the above result is valid for any compact Lie group. Indeed, if we start with the self-duality equations (2.9), written in the equivalent form ${ }^{3}$

$$
* F^{a \mu \nu}=-\frac{1}{2 \sqrt{-g}} \varepsilon^{\mu \nu \rho \lambda} F_{\rho \lambda}^{a}=i F^{a \mu \nu},
$$

then we have

$$
\frac{i}{\sqrt{-g}} \partial_{\mu}\left(\sqrt{-g} F^{a \mu \nu}\right)=-\frac{1}{2 \sqrt{-g}}\left(\varepsilon^{\mu \nu \rho \lambda} \partial_{\mu} F_{\rho \lambda}^{a}\right) .
$$

But, it can be verified by a direct calculus that the following property is satisfied if the tensor $F_{\rho \lambda}^{a}$ is self-dual:

$$
\frac{1}{2 \sqrt{-g}}\left(\varepsilon^{\mu \nu \rho \lambda} \partial_{\mu} F_{\rho \lambda}^{a}\right)=i f_{b c}^{a} A_{\mu}^{b} F^{c \mu \nu} .
$$

Now, if we introduce (4.12) into (4.11) then we obtain the Yang-Mills equations (4.2). Therefore, from the self-duality equations (of the first order) one obtains the Yang-Mills equations (of the second order) for any gauge theory with compact Lie group.

\section{Concluding remarks}

In this paper we have considered a spherically symmetric $\mathrm{SU}(2)$ gauge theory in a curved spacetime. The self-duality equations for the gauge fields have been obtained and the connection with the Yang-Mills equations has been investigated. We proved that Yang-Mills equations result from duality and that this property is true for any compact Lie group.

The Einstein equations for the metric coefficients can not be obtained from self-duality. They should be derived if we would consider a gauge theory with the direct product group $P \times S U(2)$, where $P$ is the Poincaré group. Because the N-extended super- groups include both the Poincaré and an internal group of symmetry like $\mathrm{SU}(\mathrm{N})$, it is important to study the selfduality equations for the supersymmetry. ${ }^{7}$

\section{Rferences}

1. M.S. Volkov, D.V. Gal'tsov, Phys.Reports 319, 1 (1999). 
2. B. Felsager, Geometry, particles and fields, Odense Univ. Press, 1981.

3. G. Zet, Self-duality equations of gauge theories (to be published).

4. F.A. Bais, R.J. Russel, Phys. Rev. D11, 2692 (1975).

5. M.Yu. Zotov, Dynamical system analysis for the Einstein-Yang-Mills equations, gr-qc/9906024.

6. G. Zet, V. Manta, Mod. Phys. Lett. A16, 685 (2001).

7. E. Sezgin, Self-duality and supersymmetry, hep-th/9212092. 